EPJ Web of Conferences 16, 03005 (2011)

DOI: $10.1051 /$ epjconf/20111603005

(C) Owned by the authors, published by EDP Sciences, 2011

\title{
XAO coronagraphy with the High-Order Test bench
}

\author{
P. Martinez ${ }^{\mathrm{a}}$, E. Aller Carpentier and M. Kasper \\ European Southern Observatory, Karl-Schwarzschild-Strasse 2, 85748 Garching, Germany
}

\begin{abstract}
Extreme adaptive optics systems dedicated to the search for extra solar planets are currently being developed for 8-10 meter class telescopes. The High-Order Test bench (HOT) is a high-contrast imaging adaptive optics bench developed at ESO. Its objective is to test and optimize different techniques and technologies (e.g. wave front sensors, coronagraphs, speckle calibration methods...). It mimics realistic conditions at a telescope (e.g. VLT), including turbulence generator, XAO, and various near-IR coronagraphs. Here we provide a short overview of the system, and present results of recent AO-closed loop coronagraphic runs obtained with an Apodized Pupil Lyot Coronagraph (APLC).
\end{abstract}

\section{THE HIGH-ORDER TEST BENCH}

HOT is combining a turbulence generator with phase screens to simulate real seeing conditions, a VLT-pupil mask installed on a Tip-Tilt mount, a 60-bimorph deformable mirror correcting for the static aberrations, a $32 \times 32$ micro deformable mirror (DM hereafter, electrostatic MEMS device) correcting for the dynamic turbulence, a beam-splitter transmitting the visible light to wave front sensing either with a Shack-Hartmann (SWS) or a pyramid concept, while the infrared light is directed towards coronagraph and infrared camera $\left(1 \mathrm{k} \times 1 \mathrm{k}\right.$ HAWAII detector, cooled at $\left.105^{\circ} \mathrm{K}\right)$. The turbulence is generated using two phase screens in reflection with a diameter of $50 \mathrm{~mm}$, rotating independently from each other, specified to reproduce a turbulence of $0.5^{\prime \prime}$ seeing (low-order reduced, required to avoid saturation of the DM which has limited actuator stroke of about two microns.). The DM actuator pitch is $340 \mu \mathrm{m}$, leading to an AO cut-off frequency of $15 \lambda / \mathrm{D}$ (i.e. $0.6^{\prime \prime}$ for a 8 -meter telescope in H-band). During the experiment, the XAO system was operating with a SWS. The SWS closed-loop runs at $80 \mathrm{~Hz}$. In $0.5^{\prime \prime}$ seeing with $1.3 \mathrm{~m} / \mathrm{s}$ wind speed, the system deliver a $90 \%$ Strehl ratio in H-band at high-flux (star magnitude of 5). Further details on HOT characteristics and optimization can be found in [1].

Several concepts of coronagraph have been developed to operate on HOT [2]: classical Lyot coronagraphs, a four-quadrants phase mask, an apodized pupil Lyot coronagraph [3], and some bandlimited coronagraphs [4]. Apart from the latter, these concepts are representative of the ones selected for SPHERE [5]. The APLC (4.5 $\lambda / \mathrm{D}$, IWA, i.e. 90 mas) apodizer is manufactured with a halftone-dot process (square-shaped opaque Chrome dots of $4.5 \times 4.5 \mu \mathrm{m}$ ). Further details of the APLC prototype can be found in [3], where it has been tested and validated in an infrared coronagraphic test bench.

\section{AO-CLOSED LOOP CORONAGRAPHIC RESULTS}

The AO-corrected and apodized PSF (Figure 1 left) reveals the diffraction pattern of the VLT-pupil owing to a high $90 \%$ Strehl ratio. The AO cut-off frequency is identified in the image with a dashed-white circle, and is localized at $15 \lambda / \mathrm{D}$, i.e. $0.6^{\prime \prime}$. The APLC image (Figure 1, middle)

\footnotetext{
ae-mail: martinez@eso.org
}

This is an Open Access article distributed under the terms of the Creative Commons Attribution-Noncommercial License 3.0, which permits unrestricted use, distribution, and reproduction in any noncommercial medium, provided the original work is properly cited. 


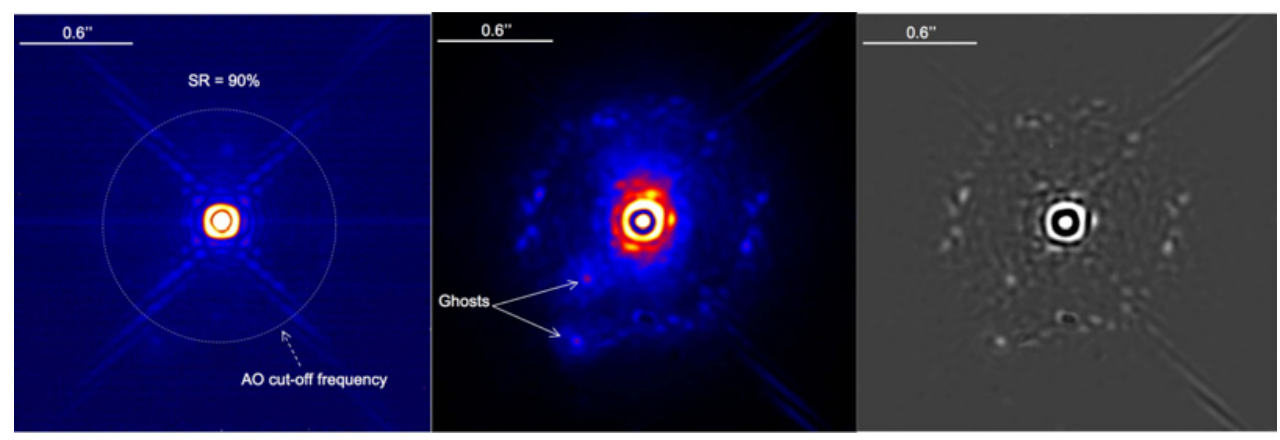

Figure 1. From left to right: AO-corrected and apodized PSF, APLC, and high-pass filtered APLC images.

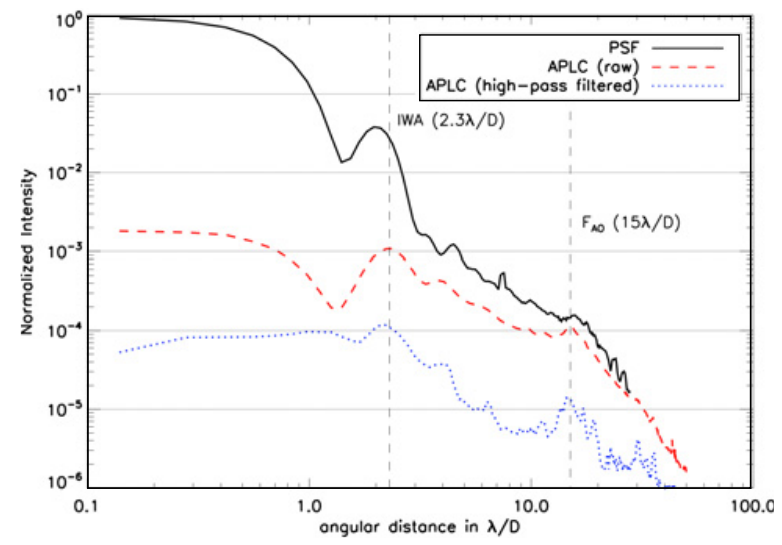

Figure 2. Azimuthally averaged contrast profiles in $\mathrm{H}$-band $(\Delta \lambda / \lambda=4 \%)$.

demonstrates starlight attenuation, and exhibits atmospheric speckles with lower intensity in the AO-correction domain, while the central part of the APLC image is dominated by diffraction residuals and pinned speckles (bright speckles localized at the position of the diffraction rings). While the dynamic atmospheric wave front has been reduced by the XAO system, to indentify the limit imposed by the speckle background, the APLC image has been high-pass filtered (Figure 1 right) to remove smooth structures of the image (atmospheric speckle halo), leaving the small scale higher frequencies components (e.g. planets) unaffected. Contrast profiles are presented in Figure 2 and nicely fulfilled SPHERE [6] expectation at similar level of contrast.

\section{References}

[1] E. Aller Carpentier, M. Kasper, P. Martinez et al., Proc. SPIE. 7015, $70153 Z$ (2008)

[2] P. Martinez, E. Vernet, C. Dorrer et al., Proc. SPIE. 7015, 70156B (2008)

[3] P. Martinez, C. Dorrer, E. Aller Carpentier et al., A\&A. 495, 363-370 (2009)

[4] P. Martinez, C. Dorrer, M. Kasper, ApJ, 705, 1637-1645 (2009)

[5] A. Boccaletti, L. Abe, J. Baudrand et al., Proc. SPIE. 7015, 70151B (2008)

[6] A. Boccaletti, M. Carbillet, T. Fusco et al., Proc. SPIE, 7015, 70156E (2008) 